\title{
COMMENT
}

\section{Comparisons between cataract surgery and aviation}

\author{
Thomas Charles Wood $\mathbb{D}^{1 凶}$, Sundas Maqsood ${ }^{1}$, William Sancha ${ }^{2}$, Mayank A. Nanavaty $\mathbb{D}^{1,3}$ and Saul Rajak ${ }^{1,3}$ \\ (c) The Author(s), under exclusive licence to The Royal College of Ophthalmologists 2021
}

Eye (2022) 36:490-491; https://doi.org/10.1038/s41433-021-01877-4

\begin{abstract}
INTRODUCTION
Ophthalmic surgery is responsible for $3.4 \%$ of all patient safety incidents in healthcare [1]. Failings in human factors (HF) and nontechnical skills (NTS) are more commonly implicated than technical skills (TS) in preventable errors such as wrong intraocular lens (IOL) events [2]. Similarly, HF and NTS failings account for $70 \%$ of aviation accidents or incidents, although advanced training and implementation of NTS within aviation has improved their safety standards and outcomes in recent decades [3-5]. Parallels are increasingly drawn between the values, culture and core beliefs of aviation and surgery, in particular with the predictable, high volume and repeated procedures [2]. Given the recognition that surgeons can learn lessons from the experiences of pilots, we explore the comparisons between aviation and cataract surgery.
\end{abstract}

\section{Repetitive events}

Cataract surgery is the most commonly performed surgical procedure in the UK, whilst 85,000 daily flights occurred before the COVID-19 pandemic $[2,6]$. Cataract surgery has similarities with short-haul flying; multiple daily repetitive events occur with pre-planned routine, standardised preparation, controlled trajectories/destinations, and are executed by highly trained teams and individuals. Each carries an established risk of relatively predictable adverse outcomes. Both are exposed to unpredictable external factors, and are carried out by fallible humans performing complex tasks using advanced and expensive equipment on a background of complex science [6].

\section{Training and assessment}

Comparisons between surgery and aviation correlate the surgeon with the pilot. High levels of training are required to achieve competence in both disciplines. Pilots obtain and maintain competence through basic flight and aircraft-specific training (during which time minimum flight hours must be met), followed by six-monthly simulator sessions and regular check flights for the remainder of their careers [7, 8]. Pilots are regularly assessed according to competencies specified by regulating bodies and individual airlines in live and simulated settings, however surgical competence is relatively informally assessed in live cataract surgery $[6,7]$. Whilst simulation in cataract surgery training has been limited and voluntary, simulation may now be of greater value than ever before as junior surgeons return from redeployment after the COVID-19 pandemic. Although flight simulators are more expensive than cataract surgery simulations, realistic cataract surgery simulation is arguably more challenging to achieve given the wide-ranging permutations of ocular, patient, environmental and staffing factors. Furthermore, aviation has formally incorporated NTS into training, simulation and assessment due to its strong associations with safety enhancement $[2,9,10]$. Pilots risk multiple fatalities (including their own) through severe NTS failures, whereas NTS failures do not threaten the cataract surgeon's vision when undertaking complicated surgery $[2,7]$.

Presently, ophthalmic surgeons undergo foundation/internship training followed by apprenticeship-based speciality training, during which time trainees must complete a minimum of 350 cataract surgeries, before achieving Consultant/Surgeon status [11]. Surgical training programmes are adopting competencybased training and assessment methods, however once 'competency' has been achieved, the surgeon's requirement for demonstrating ongoing proficiency is negligible compared to pilots (depending on the requirements of the regulating professional body) $[7,12]$. Fully qualified ophthalmic surgeons and pilots do not have minimum case number or flight hour requirements in order to retain their licences, however pilots are required to demonstrate specific skills (such as take-offs and landings) after extended breaks from flying ('recency') [6]. Prior to recommencing live surgery, there are no comparable requirements for ophthalmic surgeons to formally demonstrate their competencies after extended breaks. This is particularly relevant given the ongoing disruption to both industries caused by the COVID-19 pandemic $[13,14]$.

\section{Adverse events, challenges and complications}

Challenging and complicated situations are encountered by pilots and cataract surgeons alike, and can result in adverse events and complications. Adverse events or unintended outcomes occur in around $5 \%$ of cataract surgeries in comparison to 3.2 incidents per million passengers in aviation $[15,16]$. Complications encountered during cataract surgery include posterior capsule rupture, zonular dehiscence and suprachoroidal haemorrhage, whilst in-flight complications include bird strikes, landing gear problems and engine failures. Complications in both disciplines may derive from technical and nontechnical factors, and must be managed in controlled, practiced and skilled manners in order to optimise flight and visual outcomes $[15,17]$.

Checklists, briefs and de-briefs are used in both disciplines to try to predict and reduce the risk and severity of complications $[5,6]$. Checklists were introduced in aviation in the 1950s following the recognition that complex procedural steps could not be reliably memorised [3]. In operating theatres, cataract surgery checklists were introduced in 2010 [6, 18]. The comparison is, however,

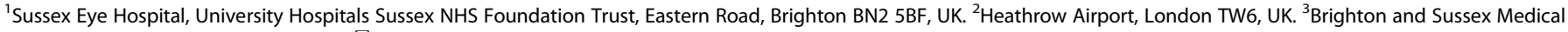
School, Falmer, Brighton BN1 9PX, UK. ${ }^{凶}$ email: tomwoodresearch@gmail.com

Received: 28 March 2021 Revised: 20 November 2021 Accepted: 25 November 2021

Published online: 3 January 2022 
imperfect. Aviation checklists enable the alignment of general flight safety requirements with standard operating procedures of specific aircraft models. Factors within the anatomical, pathological and physiological domains of the patient cannot be standardised to the same degree in surgical checklists [9].

\section{Technology and equipment}

The complex equipment used in aviation and cataract surgery are not comparably standardised, and the ability to operate them may vary. Pilot's workload management benefits from highly standardised and automated monitoring and alerting systems, unlike in cataract surgery where the surgeon carries greater responsibility of patient monitoring and technical activities. Cataract surgery has more opportunities for intra-procedural abortion than aviation; an aircraft must commit to take-off after passing V1 (the speed at which there is insufficient runway remaining to allow the aircraft to stop). Pilots must know the function of every button in the cockpit to which they were trained, with additional support available including manuals and radio advice from the ground [19]. Cataract surgeons may not know the intricacies of various machines when working at different hospitals, and may need to rely on supporting staff for initiating appropriate setting selections [20]. Furthermore, a single aircraft model allows for absolute standardisation in the way that one uses it, whereas no two patients undergoing cataract surgery will be anatomically or physiologically identical [9].

\section{Culture, working conditions and environment}

Variation in the working conditions, interpersonal dynamics and blame cultures of pilots and cataract surgeons exist. A minimum of two pilots are required at the flight deck at all times, whereas there is commonly only one operating surgeon for multiple patients on a single list. To prevent tiredness and fatigue, pilots have limitations on flight time and working hours, and must take breaks on longhaul flights $[19,21]$. There are no requirements for breaks during high volume cataract surgery lists; self-regulation is the primary safeguard $[5,21]$. Pilots are required to have a low threshold to admit when they do not feel fit to fly, whereas this culture is uncommon amongst surgeons [21]. The 'just culture' in aviation champions openness about errors and near misses, in order to promote learning and avoid dangerous repetitions [7]. A comparable system does not exist within cataract surgery, for reasons including reputational damage and litigation anxieties [22].

\section{CONCLUSION}

Whilst the comparisons are inexhaustive and imperfect, cataract surgeons can learn vital lessons from understanding the similarities and differences between cataract surgery and aviation. The global discipline of aviation promotes international efforts to standardise practices, procedures and training methods, but differences in international economics, cultures and healthcare provision inhibits comparable standardisation of these aspects in cataract surgery [7]. Through the implementation of cultural and training practices employed in aviation, cataract surgery stands to enhance safety standards and patient outcomes.

\section{REFERENCES}

1. Catchpole K, Panesar SS, Russell J, Tang V, Hibbert P, Cleary K. Surgical safety can be improved through better understanding of incident reported to a national database. Nat Patient Safety Agency. 2009.

2. Azuara-Blanco A, Reddy A, Wilkinson G, Flin R. Safe eye surgery: non-technical aspects. Eye. 2011;25:1109-11.

3. Gerstle CR. Parallels in safety between aviation and healthcare. J Pediatr Surg. 2018;53:875-8
4. Steeples LR, Hingorani M, Flanagan D, Kelly SP. Wrong intraocular lens events-what lessons have we learned? A review of incidents reported to the National Reporting and Learning System: 2010-2014 versus 2003-2010. Eye. 2016;30:1049-55.

5. Brennan PA, Mitchell DA, Holmes S, Plint S, Parry D. Good people who try their best can have problems: recognition of human factors and how to minimise error. Br J Oral Maxillofac Surg. 2016;54:3-7.

6. Singh N. On a wing and a prayer: surgeons learning from the aviation industry. J R Soc Med. 2009;102:360-4.

7. Sommer KJ. Pilot training: What can surgeons learn from it? Arab J Urol. 2014;12:32-5.

8. Todd MA, Thomas MJ. Experience, competence, or syllabus? Influences on flight hours at licensing of commercial pilots. Int J Aviat Psychol. 2013;23:169-80.

9. Isreb S, Attwood SE. The fallacy of comparing surgeons with pilots in the search for safer surgical training. J Br Surg. 2011;98:467-8.

10. Wood TC, Maqsood S, Nanavaty MA, Rajak S. Validity of scoring systems for the assessment of technical and non-technical skills in ophthalmic surgery-a systematic review. Eye. 2021;35:1833-49.

11. Turnbull AMJ, Lash SC. Confidence of ophthalmology specialist trainees in the management of posterior capsule rupture and vitreous loss. Eye. 2016;30:943-8.

12. Benjamin L. Selection, teaching and training in ophthalmology. Clin Exp Ophthalmol. 2005;33:524-30.

13. Lin PF, Naveed $H$, Eleftheriadou M, Purbrick R, Ghanavati MZ, Liu C. Cataract service redesign in the post-COVID-19 era. Br J Ophthalmol. 2021;105:745-50.

14. Gössling S. Risks, resilience, and pathways to sustainable aviation: a COVID-19 perspective. J Air Transp Manag. 2020;89:101933.

15. Chan E, Mahroo OAR, Spalton DJ. Complications of cataract surgery. Clin Exp Optom. 2010;93:379-89.

16. Pal A, Lal R, Frizelle F. Aviation-based teamwork skills work for surgeons: time for an "aviation bundle"? ANZ J Surg. 2018;88:1231-5.

17. Yorston D, Duncalfe M. Managing emergencies: lessons from aviation. Community Eye Health. 2018;31:58.

18. Kelly SP, Steeples LR, Smith R, Azuara-Blanco A. Surgical checklist for cataract surgery: progress with the initiative by the Royal College of Ophthalmologists to improve patient safety. Eye. 2013;27:878-82.

19. Schwaitzberg SD, Godinez C, Kavic SM, Sutton E, Worthington RB, Colburn B, et al. Training and working in high-stakes environments: lessons learned and problems shared by aviators and surgeons. Surgical Innov. 2009;16:187-95.

20. Wood TC, Maqsood S, Zoutewelle S, Nanavaty MA, Rajak S. Development of the HUman Factors in intraoperative Ophthalmic Emergencies Scoring System (HUFOES) for non-technical skills in cataract surgery. Eye. 2021;35:616-24.

21. Aerden D, Smets D, Poelaert J, Oste J, Brande PVD. Fighting human error: what surgeons can learn from aviators. Acta Chirurgica Belgica. 2014;114:228-32.

22. O'Connor T, Papanikolaou V, Keogh I. Safe surgery, the human factors approach. Surgeon. 2010;8:93-5.

\section{AUTHOR CONTRIBUTIONS}

TCW contributed to the article's concept and design, as well as drafting and revision of the manuscript. SM contributed to the article's concept and design, drafting and revision of the manuscript, provided regular supervision, and approved the final manuscript. WS contributed to revision of the manuscript, provided regular supervision, provided technical and non-technical advice from an aviation perspective, and approved the final manuscript. MAN contributed to the article's concept and design, drafting and revision of the manuscript, provided regular supervision, and approved the final manuscript. SR contributed to the article's concept and design, drafting and revision of the manuscript, provided regular supervision and approved the final manuscript.

\section{COMPETING INTERESTS}

The authors declare no competing interests.

\section{ADDITIONAL INFORMATION}

Correspondence and requests for materials should be addressed to Thomas Charles Wood.

Reprints and permission information is available at http://www.nature.com/ reprints

Publisher's note Springer Nature remains neutral with regard to jurisdictional claims in published maps and institutional affiliations. 